\title{
Heavy elements abundances in turn-off stars and early subgiants in NGC $6752^{\star}$
}

\author{
G. James $^{1}$, P. François ${ }^{1}$, P. Bonifacio ${ }^{2}$, A. Bragaglia ${ }^{3}$, E. Carretta ${ }^{4}$, M. Centurión ${ }^{2}$, G. Clementini ${ }^{3}$, S. Desidera ${ }^{5,6}$, \\ R. G. Gratton ${ }^{4}$, F. Grundahl ${ }^{7}$, S. Lucatello ${ }^{4,6}$, P. Molaro ${ }^{2}$, L. Pasquini ${ }^{8}$, C. Sneden ${ }^{9}$, and F. Spite ${ }^{1}$ \\ 1 Observatoire de Paris - GEPI, 61 avenue de l'Observatoire, 75014 Paris, France \\ 2 Osservatorio Astronomico di Trieste, via G. B. Tiepolo 11, 34131 Trieste, Italy \\ 3 Osservatorio Astronomico di Bologna, via Ranzani 1, 40127 Bologna, Italy \\ ${ }^{4}$ Osservatorio Astronomico di Padova, Vicolo dell'Osservatorio 5, 35122 Padova, Italy \\ 5 Dipartimento di Fisica, Università di Pisa, Piazza Torricelli 2, 56100 Pisa, Italy \\ ${ }^{6}$ Dipartimento di Astronomia, Università di Padova, Vicolo dell'Osservatorio 2, 35122 Padova, Italy \\ 7 Department of Astronomy, University of Aarhus, Bygning 520, 8000 Aarhus C, Denmark \\ ${ }^{8}$ European Southern Observatory, Karl-Schwarzschild-Str. 2, 85748 Garching bei München, Germany \\ 9 Department of Astronomy, University of Texas at Austin, RLM 15.308, C-1400, Austin, TX, USA
}

Received 26 June 2003 / Accepted 28 October 2003

\begin{abstract}
High resolution spectra $(R \geq 40000)$ for 9 main sequence turn-off stars and 9 subgiants in the globular cluster NGC 6752 were acquired with UVES on the VLT-Kueyen (UT2). These data have been used to determine the abundances of some heavy elements (strontium, yttrium, barium and europium). This paper presents for the first time accurate results for heavy elements in this globular cluster. We did not find any systematic effect between the abundances found in turn-off stars, subgiants, and giants. We obtain the following mean abundances for these elements in our sample (turn-off stars and subgiants): $[\mathrm{Sr} / \mathrm{Fe}]=0.06 \pm 0.16,[\mathrm{Y} / \mathrm{Fe}]=-0.01 \pm 0.12,[\mathrm{Ba} / \mathrm{Fe}]=0.18 \pm 0.11$, and $[\mathrm{Eu} / \mathrm{Fe}]=0.41 \pm 0.09$. The dispersion in the abundance ratios of the different elements is low and can be totally explained by uncertainties in their derivation. These ratios are in agreement with the values found in field halo stars with the same metallicity. We did not observe any correlation between the $[\mathrm{n}$-capture/Fe] ratios and the star-to-star variations of the $\mathrm{O}$ and $\mathrm{Na}$ abundances. The $[\mathrm{Ba} / \mathrm{Eu}]$ and $[\mathrm{Sr} / \mathrm{Ba}]$ ratios show clearly that this globular cluster has also been uniformly enriched by $\mathrm{r}$ - and s-process synthesis.
\end{abstract}

Key words. stars: abundances - Galaxy: globular clusters: individual: NGC 6752

\section{Introduction}

Spectroscopic observations of globular cluster stars can provide many clues to understand the formation and evolution of the early Galaxy. As they are among the oldest stellar objects known, they can be used as natural laboratories for testing the theory of stellar evolution.

In a given Galactic globular cluster, stars are commonly accepted to have all about the same age and at least the same metallicity. But if there doesn't seem to be evidence yet for any kind of age variation among stars in a single cluster, small scatters in their individual abundances have already been found, thus leading to the question of the primordial or evolutionary origin of these scatters. Most globular clusters (GCs) seem to have a rather homogeneous iron abundance, but there is evidence of a few clusters with star-to-star variations in the

Send offprint requests to: G. James,

e-mail: Gael.James@obspm.fr

* Based on observations made with the ESO VLT-UT2 at the Paranal Observatory, Chile (ESO-LP 165.L-0263). abundances of iron-peak elements (e.g. $\omega$ Cen). Further striking star-to-star variations are also seen in the abundance patterns of other elements (e.g. $\mathrm{CH}$ and $\mathrm{CN}$ bands, $\mathrm{Al}, \mathrm{Na}$, and $\mathrm{Mg}$ variations, and $\mathrm{Al}-\mathrm{O}$ or $\mathrm{O}-\mathrm{Na}$ anticorrelations). The detection of heavier elements and the study of their abundance patterns can be used as a tool to understand the origin of the chemical enrichment of a globular cluster. Especially, a comparison of the abundances of neutron-capture elements (roughly $A>60$ ) can be very helpful to distinguish which chemical enrichment scenario has more probably taken place among the cluster stars, depending on the relative fraction of elements produced by the $r$ - or the s-process. As almost every globular cluster chemical analysis up to now has only been done on giant stars, it is therefore also significant to investigate the possible inhomogeneity of the abundances in the different evolutionary sequences of a globular cluster.

This work is part of the ESO-Large Program 165.L-0263 (PI: R. G. Gratton), which is dedicated to the analysis of highresolution spectra $(R \geq 40000)$ of a large sample of dwarfs near the turn-off and early subgiants at the base of the red 
giant branch (RGB) in at least three globular clusters (47 Tuc, NGC 6397 and NGC 6752). The first results of this program (Gratton et al. 2001) already showed clearly - and for the first time - the $\mathrm{O}-\mathrm{Na}$ anticorrelation among turn-off stars and early subgiants in NGC 6752, ruling out the hypothesis of mixing as being the origin of these anomalies. A more recent work has been done by Bonifacio et al. (2002) on the lithium content of NGC 6397, its possible primordial origin in this cluster, and its influence on the standard big-bang model.

Previous precise abundance determinations in globular clusters have only been done for bright giant stars $(V \sim 11-12)$, and the few attempts to derive abundances in fainter or less evolved stars (e.g. Boesgaard et al. 1998; King et al. 1998) obtained only very low $S / N$ spectra, and thus not really reliable measurements of the n-capture elements. UVES, the echellespectrograph at the VLT-UT2 (Kueyen), is one of the few instruments able to obtain spectra with higher $S / N$ values for such faint stars $(V \sim 16-17)$, which allows to measure accurate abundances also for heavy elements.

Concerning the cluster NGC 6752, there are only a few references about abundance determinations, most of them being metallicity (in this case, iron abundances) determinations (Zinn \& West 1984; Minniti et al. 1993; Carretta \& Gratton 1997), and some others beeing abundance analyses including several other elements (Norris \& Da Costa 1995; Grundahl et al. 2002; Yong et al. 2003). All these studies have been done on bright giant stars, and the only few results on heavy elements are reported in the paper of Norris \& Da Costa (1995).

In this paper, we present for the first time accurate results for abundances of several heavy elements ( $\mathrm{Sr}, \mathrm{Y}, \mathrm{Ba}$, and $\mathrm{Eu}$ ) in turn-off stars and early subgiants (at the base of the RGB) in a globular cluster, NGC 6752.

\section{Observations}

The observations were carried out in several runs at the Kueyen (VLT-UT2) Telescope with the UVES high-resolution echelle spectrograph in June and September 2000, and they have already been described by Gratton et al. (2001). For the cluster NGC 6752, data have been obtained for 9 dwarfs around the turn-off (TO) point, and 9 subgiants at the base of the RGB.

The observed stars are listed in Table 1. Star identifications and photometry are from Grundahl et al. (2000). Exposure times range from about 1 hour for the brightest subgiants to about 4 hours (split in 3 to 4 exposures) for the faintest turn-off stars. The observations were done with the UVES Dichroic \#2 mode, which allows to cover a wide spectral range (350-470 $\mathrm{nm}$ for the blue spectra, and 570-870 $\mathrm{nm}$ for the red ones). The resolution $(R \equiv \lambda / \Delta \lambda)$ is always over 40000 , depending mainly on the slit width (see Gratton et al. 2001).

In Table 1, we give $S / N$ ratios per pixel around 450 and $650 \mathrm{~nm}$ (there are $\sim 5$ pixels per resolution element), near the observed lines of strontium, yttrium, barium, and europium (Sr II: 407.77 and $421.55 \mathrm{~nm}$; Y II: 395.03 and $439.80 \mathrm{~nm}$; Ba II: 455.40, 585.37, 614.17 and 649.69 nm; Eu II: 412.97 and $420.50 \mathrm{~nm}$ ). $S / N$ ratios range from $\sim 15$ in the worst cases of the blue spectra (for the turn-off star \#4661), to $\sim 65$ in the red spectra (subgiant \#1400, and turn-off star \#4907). Figure 1 shows
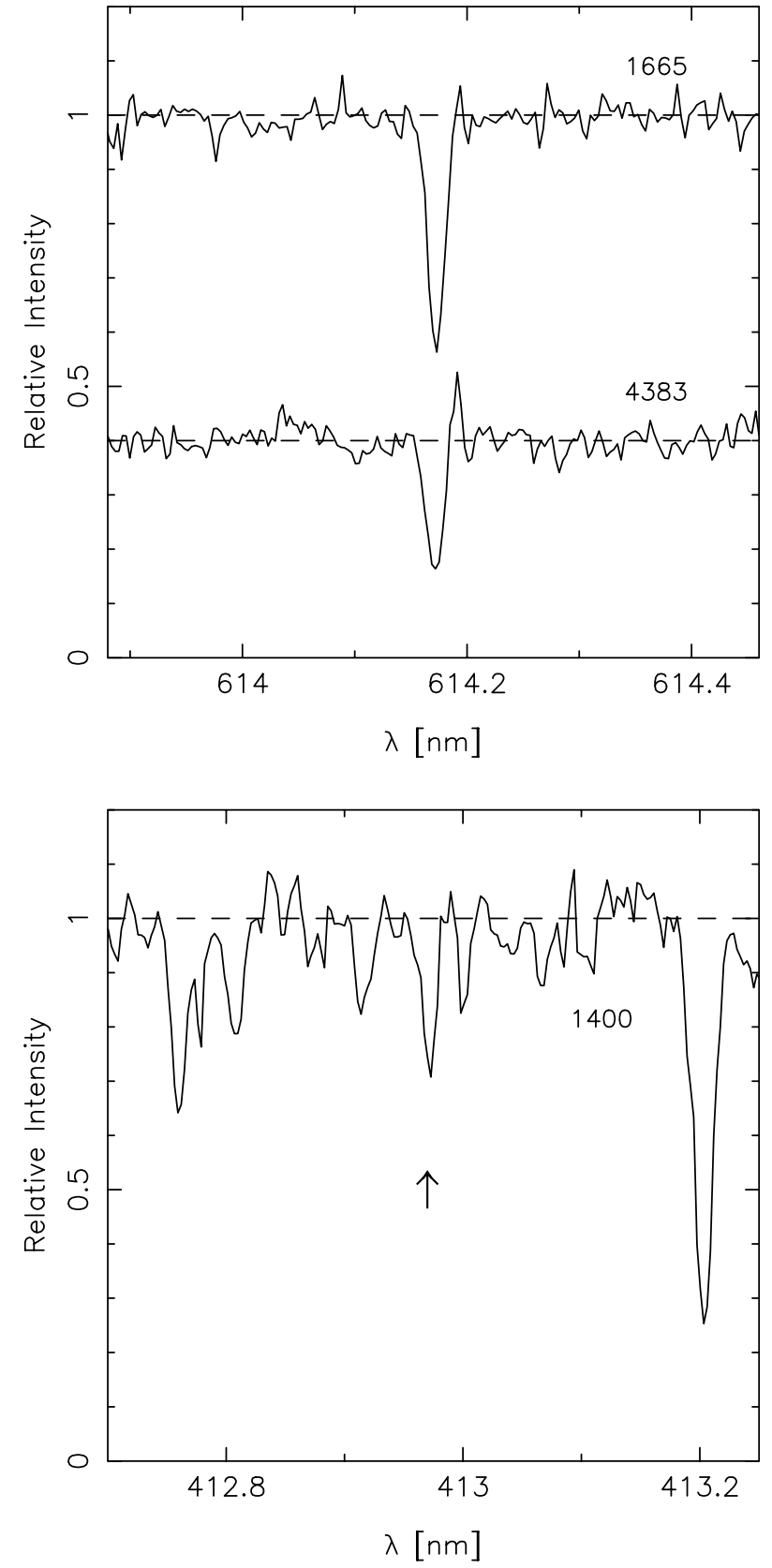

Fig. 1. Top: UVES (VLT-UT2) normalized spectra of NGC 6752 stars \#1665 (subgiant) and \#4383 (dwarf), centered on the Ba II (614.17 nm) line, showing a direct comparison of the strength of the lines between subgiants and dwarfs in the cluster. The spectrum of star \#4383 has been offsetted to avoid overlap. Bottom: spectral region of star \#1400 (subgiant), centered on the Eu II (412.97 nm) line.

typical examples of our spectra in the regions of the Ba II line at $614.17 \mathrm{~nm}$, and the Eu II line at $412.97 \mathrm{~nm}$.

\section{Abundance analysis}

\subsection{Model atmospheres and stellar parameters}

The adopted model atmospheres (OSMARCS, LTE) have been computed using the grid defined by Edvardsson et al. (1993), with an updated version of the MARCS code of Gustafsson et al. (1975) with improved UV-line blanketing (see also 
Table 1. Data for our NGC 6752 Stars. Star identifications and photometry are from Grundahl et al. (2000).

\begin{tabular}{|c|c|c|c|c|c|c|c|c|c|}
\hline Star \# & $V$ & $b-y$ & $\begin{array}{c}S / N \\
@ 450 \mathrm{~nm}\end{array}$ & $\begin{array}{c}S / N \\
@ 650 \mathrm{~nm}\end{array}$ & {$[\mathrm{Fe} / \mathrm{H}]$} & {$[\mathrm{Sr} / \mathrm{Fe}]$} & {$[\mathrm{Y} / \mathrm{Fe}]$} & {$[\mathrm{Ba} / \mathrm{Fe}]$} & {$[\mathrm{Eu} / \mathrm{Fe}]$} \\
\hline \multicolumn{10}{|c|}{$\begin{array}{c}\text { Subgiants } \\
\left(T_{\mathrm{eff}}=5347 \mathrm{~K} ; \log g=3.54 ;[\mathrm{Fe} / \mathrm{H}]=-1.50 ; \xi=1.1 \mathrm{~km} \mathrm{~s}^{-1}\right)\end{array}$} \\
\hline 1400 & 15.89 & 0.50 & 49 & 65 & -1.40 & -0.12 & 0.00 & 0.10 & 0.37 \\
\hline 1406 & 15.90 & 0.48 & 54 & 58 & -1.47 & 0.03 & -0.02 & 0.20 & 0.42 \\
\hline 1445 & 15.89 & 0.50 & 36 & 57 & -1.56 & 0.00 & -0.09 & 0.33 & 0.51 \\
\hline 1460 & 15.94 & 0.47 & 35 & 58 & -1.42 & -0.04 & -0.23 & 0.20 & 0.31 \\
\hline 1461 & 15.95 & 0.49 & 26 & 44 & -1.40 & -0.07 & -0.10 & 0.23 & 0.25 \\
\hline 1481 & 15.95 & 0.46 & 42 & 62 & -1.52 & 0.13 & 0.00 & 0.30 & 0.46 \\
\hline 1563 & 16.03 & 0.47 & 33 & 52 & -1.60 & 0.06 & 0.22 & 0.36 & 0.54 \\
\hline 1665 & 16.04 & 0.48 & 30 & 47 & -1.50 & 0.07 & 0.03 & 0.27 & 0.36 \\
\hline 202063 & 15.94 & 0.47 & 27 & 50 & -1.55 & -0.11 & 0.13 & 0.23 & 0.40 \\
\hline$<$ mean $>$ & & & & & -1.49 & -0.01 & -0.01 & 0.25 & 0.40 \\
\hline$\sigma$ (std. dev.) & & & & & 0.07 & 0.09 & 0.13 & 0.08 & 0.09 \\
\hline \multicolumn{10}{|c|}{$\begin{array}{c}\text { Dwarfs } \\
\left(T_{\mathrm{eff}}=6226 \mathrm{~K} ; \log g=4.28 ;[\mathrm{Fe} / \mathrm{H}]=-1.50 ; \xi=0.7 \mathrm{~km} \mathrm{~s}^{-1}\right)\end{array}$} \\
\hline 4341 & 17.15 & 0.35 & 20 & 44 & -1.48 & -0.07 & -0.13 & 0.05 & $<0.52$ \\
\hline 4383 & 17.11 & 0.36 & 39 & 59 & -1.45 & 0.11 & 0.00 & 0.14 & $<0.44$ \\
\hline 4428 & 17.14 & 0.37 & 42 & 61 & -1.59 & 0.30 & $<0.00$ & 0.20 & $<0.63$ \\
\hline 4458 & 17.16 & 0.34 & 24 & 45 & -1.50 & -0.19 & $<0.03$ & 0.15 & $<0.62$ \\
\hline 4661 & 17.22 & 0.34 & 16 & 26 & -1.42 & 0.28 & $<0.12$ & -0.01 & $<0.67$ \\
\hline 4907 & 17.20 & 0.35 & 52 & 66 & -1.52 & 0.29 & 0.09 & 0.18 & 0.53 \\
\hline 5048 & 17.28 & 0.35 & 36 & 62 & -1.47 & 0.27 & 0.07 & 0.14 & 0.41 \\
\hline 200613 & 17.20 & 0.38 & 19 & 34 & -1.35 & 0.19 & -0.19 & -0.03 & $<0.44$ \\
\hline 202316 & 17.28 & 0.35 & 35 & 51 & -1.53 & -0.06 & 0.01 & 0.21 & $<0.47$ \\
\hline$<$ mean $>$ & & & & & -1.48 & 0.12 & -0.03 & 0.11 & 0.47 \\
\hline$\sigma$ (std. dev.) & & & & & 0.07 & 0.19 & 0.11 & 0.09 & 0.08 \\
\hline
\end{tabular}

Edvardsson et al. 1994). In these computations, we used the solar abundances of Grevesse \& Sauval (2000).

Our sample stars near the turn-off are all very close to each other in the Strömgren color-magnitude diagram of NGC 6752, and so are also the sample stars at the base of the RGB. All the stars in each one of these two sets of data have consequently very similar stellar parameters: effective temperatures $\left(T_{\text {eff }}\right)$, surface gravities $(\log g)$, and microturbulent velocities $(\xi)$. Gratton et al. (2001) already showed clearly that we could use two sets of mean atmospheric parameters: one for the TO stars, and another one for the subgiants. In the present work, we used these mean parameters, and adapted them to fit the data with our models.

To check the parameters given by Gratton et al. (2001), we first used two spectra: the average of the 9 TO stars, and the average of the 9 subgiants. The $S / N$ ratios (per pixel) of these summed spectra are much higher than those of the single spectra ( $\sim 60$ and $\sim 80$ for the blue TO and subgiants average spectra around $450 \mathrm{~nm} ; \sim 100$ and $\sim 140$ for the red TO and subgiants average spectra near $650 \mathrm{~nm}$; see Table 1 for the individual $S / N$ ratios), which allows an accurate equivalent width measurement of many reliable Fe I and Fe II lines.

Gratton et al. (2001) obtained their effective temperatures using Strömgren photometry from Grundahl et al. (2000), and by fitting the wings of the $\mathrm{H}_{\alpha}$ profiles of the spectra. They compared the resulting effective temperatures to a calibration based on the work of Alonso et al. (1996) for MS-stars. In this work, the effective temperature for our two resulting spectra was checked by assuming that there is no trend between the Fe I equivalent widths and the excitation potentials, and by fitting the wings of the $\mathrm{H}_{\alpha}$ profile of the spectra. We also checked the gravities and microturbulent velocities by comparing theoretical curves of growth with observational curves of growth. The gravities were verified with the ionization equilibrium of Fe I and Fe II, and the microturbulent velocities were checked imposing the Fe I abundances to be independent of the equivalent width of the lines. Finally, we found that the parameters used by Gratton et al. (2001) were compatible with these verifications within the error bars given in that paper.

These sets of parameters were then used to recompute the Fe abundance ${ }^{1}$ with our OSMARCS code, and we found $[\mathrm{Fe} / \mathrm{H}]=-1.50$, which we finally used as input parameter in our detailed computations.

\footnotetext{
1 We adopt here the usual spectroscopic notations that $[\mathrm{A} / \mathrm{B}] \equiv$ $\log _{10}\left(N_{\mathrm{A}} / N_{\mathrm{B}}\right)-\log _{10}\left(N_{\mathrm{A}} / N_{\mathrm{B}}\right)_{\odot}$, and that $\log \epsilon(\mathrm{A}) \equiv \log _{10}\left(N_{\mathrm{A}} / N_{\mathrm{H}}\right)+$ 12.0 , for elements $\mathrm{A}$ and $\mathrm{B}$. We assume also that metallicity is equivalent to the stellar $[\mathrm{Fe} / \mathrm{H}]$ value.
} 
As a next step, we checked for each single star that these parameters were giving coherent results by computing the abundances of up to $\sim 30$ lines of Fe I and Fe II weaker than $100 \mathrm{~mA}$. At last, we adopted the following two sets of parameters: $\left\{T_{\text {eff }}=5347 \mathrm{~K} ; \log g=3.54 ;[\mathrm{Fe} / \mathrm{H}]=-1.50 ; \xi=1.1 \mathrm{~km} \mathrm{~s}^{-1}\right\}$ for the subgiants, and $\left\{T_{\text {eff }}=6226 \mathrm{~K} ; \log g=4.28 ;[\mathrm{Fe} / \mathrm{H}]=\right.$ $\left.-1.50 ; \xi=0.7 \mathrm{~km} \mathrm{~s}^{-1}\right\}$ for the turn-off stars (see Table 1 ).

\subsection{Iron abundances}

For iron lines, we deduced abundances from equivalent width measurements made with an automatic line fitting procedure based on the algorithms of Charbonneau (1995), which perform both line detection and Gaussian fits of unblended lines. Although many more lines are visible on the spectra, we made a selection of 30-40 (depending on the quality of the spectra) unblended Fe I lines with equivalent widths lower than $100 \mathrm{~m} \AA$, and we kept all the detected unblended Fe II lines. The results for individual stars are listed in Table 1. The iron abundances are the average of the Fe I and Fe II abundances.

Previous iron abundance determinations have already been done for this cluster. For bright giant stars, Zinn \& West (1984), Minniti et al. (1993), and Norris \& Da Costa (1995) found respectively $[\mathrm{Fe} / \mathrm{H}]=-1.54,[\mathrm{Fe} / \mathrm{H}]=-1.58$ (3 stars), and $[\mathrm{Fe} / \mathrm{H}]=-1.54$ (6 stars), while Carretta \& Gratton (1997) found a higher value for their 4 observed giants $([\mathrm{Fe} / \mathrm{H}]=$ -1.42). Gratton et al. (2001) confirm this metallicity for our sample $([\mathrm{Fe} / \mathrm{H}]=-1.42)$. More recently, Grundahl et al. (2002) and Yong et al. (2003) published a metallicity of $[\mathrm{Fe} / \mathrm{H}]=-1.62$, respectively for 21 RGB bump stars, and 20 bright giant stars in this cluster. The average value of $[\mathrm{Fe} / \mathrm{H}]=-1.49 \pm 0.07$ (standard deviation around the mean value, see Table 1) for our whole sample is fully compatible with all these previous values within the errors that could have been made on our atmospheric parameters (see Sect. 3.4 hereafter).

The differences in iron abundance between the different previous analyses on this cluster have been discussed by Kraft \& Ivans (2003) and seem to be due mainly to differences in the adopted models (MARCS, Kurucz...). And more precisely, concerning the slight difference $(0.07 \mathrm{dex})$ of our value and the previous metallicity published by Gratton et al. (2001) from the same data, it is partly due to our different $T(\tau)$ laws (temperature variation as a function of the optical depth), leading to a difference in the temperature at the depth of line formation, and finally to a small shift in the metallicity (we used OSMARCS models, while the previous analysis has been done using Kurucz models). Moreover, when comparing our $\mathrm{Fe}$ abundances with those obtained in Gratton et al. (2001), it should be noted that these last are the abundances from Fe I alone. Those obtained from Fe II lines are a bit lower ( -1.49 for the TO stars, and -1.57 for the stars at the base of the RGB). If the same average than in the present paper is done, the difference between the two determinations would be very small ( $\sim 0.02 \mathrm{dex}$; the exact value depends on the weights given when computing the average). However, it should also be noticed that the value used in Gratton et al. (2001) is that appropriate for the purpose of obtaining the ages (Gratton et al. 2004) because also for the field stars we only consider Fe I.

But this is not a critical point in this work because we are presenting abundances ratios. Apart from that, we confirm the small dispersion in iron abundance: the standard deviation ${ }^{2}$ for the whole sample (TO stars and subgiants) is 0.07 (we obtain 0.10 from the data published in Gratton et al. 2001).

\subsection{Heavy elements abundances}

Strontium and yttrium abundances were computed from equivalent width measurements in the same way as our iron abundances. The excitation potentials and $\log g f$ for the Sr II and Y II lines were taken from Sneden et al. (1996). As barium and europium transitions are generally affected by hyperfine structure (hereafter hfs; e.g. see McWilliam et al. 1995; McWilliam 1998, and references therein), we computed a synthetic spectrum over several $\AA$ and compared it interactively to the observed one. We used the latest version of the synthetic spectrum codes of Spite (1967). Figure 2 shows typical comparisons between synthetic and observed spectra for the Ba II line at $614.17 \mathrm{~nm}$ and for the Eu II line at $412.97 \mathrm{~nm}$ for dwarfs and/or subgiants of our sample. The hfs parameters for the Ba II and Eu II lines have been taken respectively from McWilliam (1998) and Lawler et al. (2001).

For strontium, mean abundances were obtained from at least one line at $407.77 \mathrm{~nm}$ or at $421.55 \mathrm{~nm}$, and in most cases from both lines. For yttrium, we could almost always detect the lines at 395.03 and $439.90 \mathrm{~nm}$ in the subgiants, and at least the line at $395.03 \mathrm{~nm}$ in the dwarfs. For barium, we had at least three lines at 455.40, 614.17 and $649.69 \mathrm{~nm}$, and sometimes also a third line at $585.37 \mathrm{~nm}$. In the case of europium, we usually could detect the two expected lines at 412.97 and $420.50 \mathrm{~nm}$ in the subgiants, but for most of the TO stars, we could only give upper limits for both lines.

The abundances of these four heavy elements are given in Table 1. We obtain the following average values (with indication of the standard deviation $):[\mathrm{Sr} / \mathrm{Fe}]=0.06 \pm 0.16,[\mathrm{Y} / \mathrm{Fe}]=$ $-0.01 \pm 0.12,[\mathrm{Ba} / \mathrm{Fe}]=0.18 \pm 0.11$, and $[\mathrm{Eu} / \mathrm{Fe}]=0.41 \pm 0.09$ for our sample stars. Figure 3 displays results for $[\mathrm{X} / \mathrm{Fe}]$ as a function of $[\mathrm{Fe} / \mathrm{H}]$, where $\mathrm{X}$ is either $\mathrm{Sr}, \mathrm{Y}, \mathrm{Ba}$, or Eu. These abundance patterns will be discussed in the following sections (see Sect. 4.1).

Our computations have been done using LTE model atmospheres. But it is clear that NLTE can affect heavy elements, in particular Ba II and Sr II lines, for metal-poor stars in the metallicity range $-1.90 \leq[\mathrm{Fe} / \mathrm{H}] \leq-1.20$ (e.g. Mashonkina et al. 1999). Detailed computations of the NLTE effects have been done respectively by Mashonkina et al. (1999) and Mashonkina \& Gehren $(2000,2001)$ for Ba II, Eu II and Sr II lines. For metallicities around -1.50 , in most of the cases the NLTE corrections do not exceed 0.15 dex in intensity. However, the intensity of these effects depends strongly on the observed line and on

\footnotetext{
${ }^{2}$ Here we call standard deviation the square root of the variance: $\sigma\left(x_{1} \ldots x_{N}\right)=\sqrt{\operatorname{Var}\left(x_{1} \ldots x_{N}\right)}$, where the variance is given by $\operatorname{Var}\left(x_{1} \ldots x_{N}\right)=\frac{1}{N-1} \sum_{i=1}^{N}\left(x_{i}-\bar{x}\right)^{2}$, and $\bar{x}$ is the mean value of $\left(x_{1} \ldots x_{N}\right)$.
} 

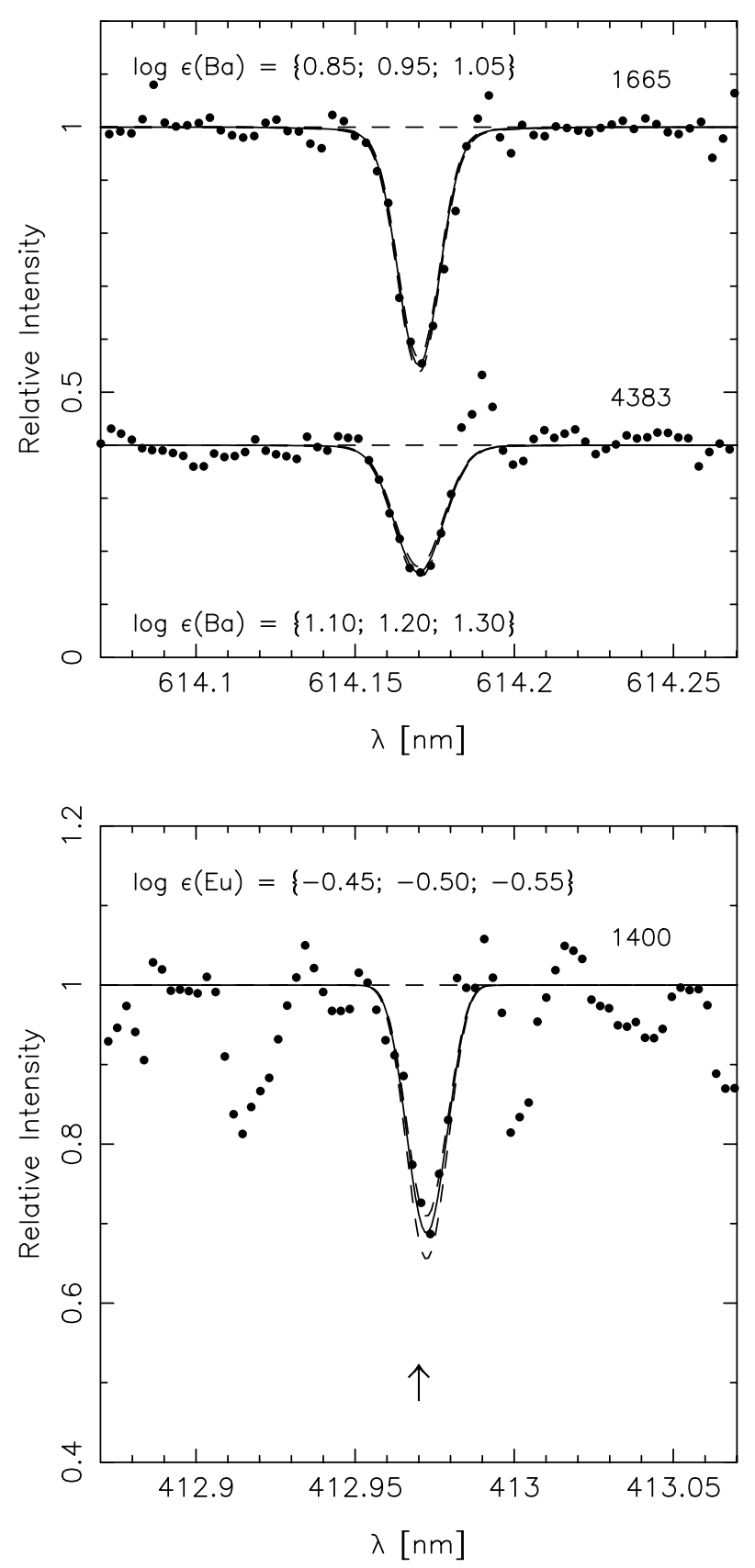

Fig. 2. Top: synthetic spectra obtained for the Ba II $(614.17 \mathrm{~nm})$ line of stars \#1665 (subgiant) and \#4383 (dwarf), with additional offset. Bottom: synthetic spectrum of the Eu II (412.97 nm) line in star \#1400 (subgiant). Dots: observations, lines: synthetic spectra.

the atmospheric parameters, and since the uncertainties on our atmospheric parameters (see Sect. 3.4 and Table 2) combined with the uncertainties on the EW measurements (or on the fits for the abundances deduced from synthetic spectra) lead to total errors of $\sim 0.15 \mathrm{dex}$, it is not certain that there would be a significant change in the abundances of our sample.

\subsection{Error estimations}

For the iron abundances, we assumed that the total error budget was due to random uncertainties in the measurement of
Table 2. Error estimates for the sample stars.

\begin{tabular}{|c|c|c|c|c|c|}
\hline El. & $N^{a}$ & $\begin{aligned} & \Delta T_{\mathrm{eff}} \\
+ & 100 \mathrm{~K}\end{aligned}$ & $\begin{array}{c}\Delta \log g \\
+0.2 \mathrm{dex}\end{array}$ & $\begin{array}{c}\Delta \xi \\
+0.2 \mathrm{~km} \mathrm{~s}^{-1}\end{array}$ & $\Delta(\operatorname{Tot})^{b}$ \\
\hline \multicolumn{6}{|c|}{ Subgiants (e.g. \#1400) } \\
\hline $\mathrm{Fe} \mathrm{I}$ & 33 & +0.09 & -0.02 & -0.05 & 0.10 \\
\hline Fe II & 11 & -0.01 & +0.07 & -0.05 & 0.09 \\
\hline Sr II & 1 & +0.04 & -0.02 & +0.02 & 0.05 \\
\hline Y II & 2 & -0.01 & +0.05 & -0.04 & 0.06 \\
\hline Ba II & 3 & +0.04 & +0.01 & -0.05 & 0.06 \\
\hline Eu II & 2 & 0.00 & +0.05 & -0.04 & 0.06 \\
\hline \multicolumn{6}{|c|}{ Dwarfs (e.g. \#4907) } \\
\hline $\mathrm{Fe} I$ & 27 & +0.09 & -0.04 & -0.05 & 0.11 \\
\hline Fe II & 8 & +0.01 & +0.06 & -0.03 & 0.07 \\
\hline Sr II & 1 & +0.05 & -0.04 & +0.01 & 0.06 \\
\hline Y II & 2 & 0.00 & +0.06 & +0.02 & 0.06 \\
\hline Ba II & 3 & +0.04 & -0.02 & -0.06 & 0.07 \\
\hline $\mathrm{Eu}$ II & 2 & 0.00 & +0.06 & +0.03 & 0.07 \\
\hline
\end{tabular}

${ }^{a}$ For Fe I, we only took into account lines with $W_{\lambda} \leq 100 \mathrm{~m} \AA$.

${ }^{b} \Delta(\mathrm{Tot})=\Delta\left(T_{\mathrm{eff}}, \log g, \xi\right)$ is the quadratic sum $\sqrt{\sum \Delta^{2}}$ of all the individual uncertainties linked to the atmospheric parameters.

the equivalent widths, and the errors made on the stellar parameters. When $N \geq 2$ lines of a given element (here Fe I or Fe II) are observed, the random uncertainties can be computed as the standard deviation around the mean abundance. The errors linked to the uncertainties on the stellar atmosphere parameters were estimated assuming the following variations: $\Delta T_{\text {eff }}= \pm 100 \mathrm{~K}, \Delta \log g= \pm 0.2 \mathrm{dex}$, and $\Delta \xi= \pm 0.2 \mathrm{~km} \mathrm{~s}^{-1}$.

For the heavy elements, as there are only a few lines detected per element, we only computed the errors for $[\mathrm{X} / \mathrm{Fe}]$ due to the uncertainties in our choice of stellar parameters. The total error budget on the stellar atmosphere parameters $\Delta\left(T_{\text {eff }}, \log g, \xi\right)$ is then the quadratic sum of the errors on the individual parameters.

Table 2 summarizes the errors estimates for one turn-off star and for one subgiant in NGC 6752. Computations of the errors for the other stars of our sample give similar results. The total error given in the last column of Table 2, slightly smaller than the scatter given in Table 1, does not take into acount the uncertainty coming from the measurements of the equivalent widths.

\section{Discussion}

\subsection{Abundance patterns}

Figure 3 displays the abundances ratios $[\mathrm{Sr} / \mathrm{Fe}],[\mathrm{Y} / \mathrm{Fe}],[\mathrm{Ba} / \mathrm{Fe}]$ and $[\mathrm{Eu} / \mathrm{Fe}]$ as a function of $[\mathrm{Fe} / \mathrm{H}]$ for our sample stars. We have added the results from Norris \& Da Costa (1995) on these figures. In order to get an homogeneous sample of data, we have recomputed their abundances in the following way. We took their published list of equivalent widths for Fe I, Fe II, Y II, Ba II and Eu II (Norris et al. 1995) and computed new 

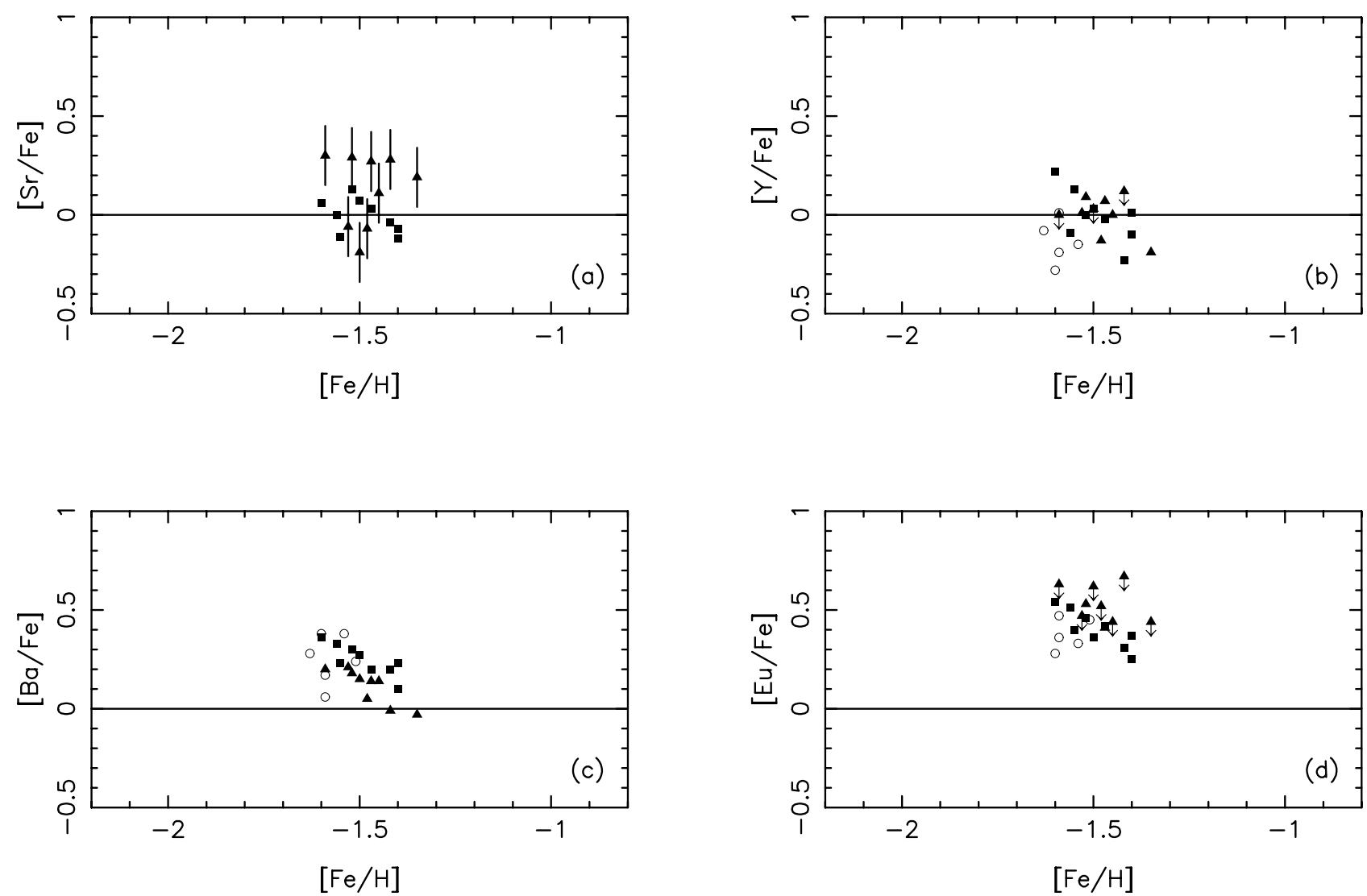

Fig. 3. $[\mathrm{X} / \mathrm{Fe}]$ ratios for the heavy elements in NGC 6752. Squares: stars at the base of the RGB. Triangles: dwarfs near the turn-off point. Arrows: upper limits for the abundances in the dwarfs. Circles: data for giants from Norris et al. (1995) and Norris \& Da Costa (1995).

abundances using our own lines parameters $(\log g f)$ and the stellar atmosphere model (OSMARCS) used for our computations. The data of Norris \& Da Costa (1995) are displayed on the figures with open circles. In the four panels, the dwarf stars are represented by triangles whereas the subgiants are represented by squares. An inspection of these figures does not seem to show large systematic differences between abundances obtained from dwarfs and subgiants.

\section{- Figure 3a: Strontium}

For strontium, we have a significant spread in the abundance ratios compared to the values found for the other elements. It is interesting to note that this effect is also found in halo field stars (e.g. see Ryan et al. 1991; Burris et al. 2000, and references therein). For dwarf stars, we have a larger spread than for subgiants (see also Table 1). This effect can be totally explained by the fact that the spectra for dwarfs are of lower quality. Although the Sr II lines are rather strong, the error on the determination of the equivalent widths comes mostly from the difficulty to estimate the continuum level. In the plot, we have added error bars for the dwarfs taking into account this additional error $(\sim 10-15 \%)$. We obtain a solar value for the mean $[\mathrm{Sr} / \mathrm{Fe}]$ ratio of $0.06 \pm 0.16$.

- Figure 3b: Yttrium

We find $[\mathrm{Y} / \mathrm{Fe}]$ ranging from -0.23 to 0.22 . There is no indication of an effect as a function of the spectral class of the star. We obtain a solar value of $-0.01 \pm 0.12$ for the mean
$[\mathrm{Y} / \mathrm{Fe}]$ ratio. This ratio is totally compatible with the values found in halo stars at intermediate metallicity (Burris et al. 2000; Fulbright 2000).

- Figure 3c: Barium

We found an overabundance of the $[\mathrm{Ba} / \mathrm{Fe}]$ ratio of +0.18 dex with a small offset $(0.14$ dex $)$ between dwarfs and subgiants. The origin of this difference remains unclear. It could partly come from the difference in the quality of the spectra. In any case, it is clear that our sample is not large enough to draw final conclusions on this matter. This result is not very different from what has been found before for this cluster (see Norris \& Da Costa 1995, who found an average value of $[\mathrm{Ba} / \mathrm{Fe}]=0.25)$, and it is also compatible with previous analyses of metal-poor stars in the same metallicity range (Burris et al. 2000; Fulbright 2000; Mashonkina et al. 2003). It is interesting to compare the present results for our almost unevolved sample of stars to the abundance ratios found in giants in other globular clusters. Sneden et al. (1997), Sneden et al. (2000), and Armosky et al. (1994) have found mean [Ba/Fe] ratios ranging from -0.29 to 0.12 in globular clusters with metallicities between -1.17 and -2.41 . Our mean value $[\mathrm{Ba} / \mathrm{Fe}]=0.18 \pm 0.11$ is in fair agreement with these values.

- Figure 3d: Europium

Europium can hardly be detected in the TO stars of NGC 6752, but the few reliable detections are compatible 


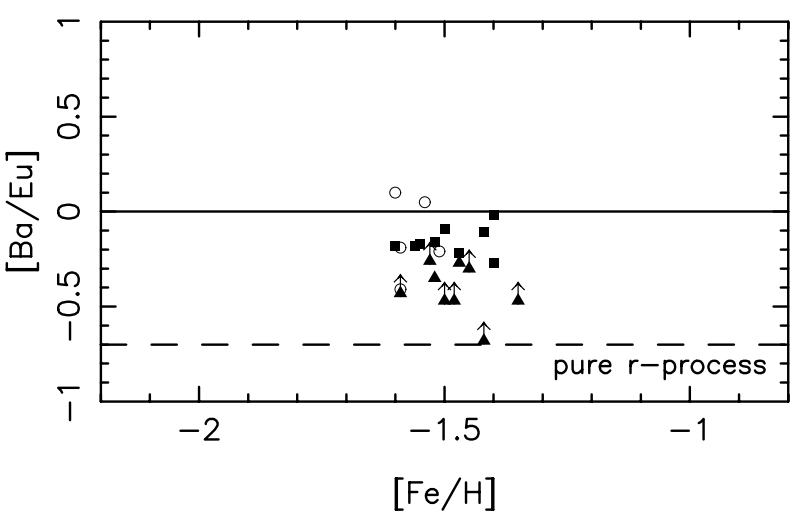

Fig. 4. $[\mathrm{Ba} / \mathrm{Eu}]$ vs. $[\mathrm{Fe} / \mathrm{H}]$. The symbols are the same than in Fig. 3.

with the abundances found in the subgiants of the cluster (see also Table 1). There is a clear overabundance of the ratio $[\mathrm{Eu} / \mathrm{Fe}]$ with a mean value of $0.41 \pm 0.09 \mathrm{dex}$. This value is in agreement with the results of Shetrone (1996) for M 71, M 5, M 13, and M 92, which are all overabundant in Eu by about the same value, and also with the ratios found in other metal-poor stars at intermediate metallicity (Burris et al. 2000; Fulbright 2000; Mashonkina et al. 2003). It is interesting to note that the dispersion of the $[\mathrm{Eu} / \mathrm{Fe}]$ ratio among the four globular clusters studied by Shetrone (1996) is much smaller than the dispersion of the $[\mathrm{Ba} / \mathrm{Fe}]$ ratio. In the present case, it is not possible to reach any conclusion concerning the different dispersion in $[\mathrm{Ba} / \mathrm{Fe}]$ and $[\mathrm{Eu} / \mathrm{Fe}]$ from our data (see Table 1).

To summarize, we did not find any large systematic difference in the abundance ratios for subgiants and turn-off stars. Our values are compatible with the abundance ratios found by Norris \& Da Costa (1995), and also with the values given by several analyses of metal-poor stars at intermediate metallicity. For the $\mathrm{Ba}$ and $\mathrm{Eu}$ abundances, the slight difference between our analysis and the paper of Norris \& Da Costa (1995) may be due to the fact that they did not consider the hyperfine structure of the transitions for these elements. No obvious correlation was detected in the comparison of the [n-capture/Fe] abundance ratios as a function of $[\mathrm{O} / \mathrm{Fe}],[\mathrm{Mg} / \mathrm{Fe}]$, and $[\mathrm{Na} / \mathrm{Fe}]$ (taken from Gratton et al. 2001).

\subsection{Neutron-capture elements ratios}

Figure 4 shows $[\mathrm{Ba} / \mathrm{Eu}]$ as a function of $[\mathrm{Fe} / \mathrm{H}]$. The horizontal full line marks the total solar abundance ratio $([\mathrm{Ba} / \mathrm{Eu}] \equiv 0)$, and the dashed line marks the solar r-process fractional ratio $[\mathrm{Ba} / \mathrm{Eu}]_{r}=-0.70$, according to Arlandini et al. (1999). The ratio $[\mathrm{Ba} / \mathrm{Eu}]$ can be used as a test of the relative importance of the s- and r-processes to the initial mix of materials at the birth of the stars. A first inspection of Fig. 4 shows a very low dispersion of the $[\mathrm{Ba} / \mathrm{Eu}]$ ratio in contrast to that found by Sneden et al. (1997). It is also interesting to note that there is no systematic difference between the ratios found for the stars in different evolutionary phases. We obtain a mean value ${ }^{3}$

\footnotetext{
3 The mean values for the $[\mathrm{Ba} / \mathrm{Eu}]$ and $[\mathrm{Sr} / \mathrm{Ba}]$ ratios haven been computed using the individual $[\mathrm{Ba} / \mathrm{Eu}]$ and $[\mathrm{Sr} / \mathrm{Ba}]$ values for each
}

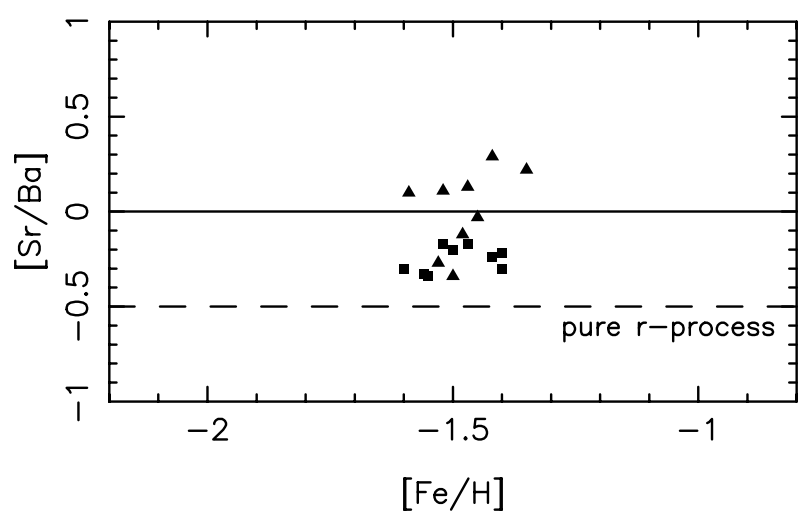

Fig. 5. $[\mathrm{Sr} / \mathrm{Ba}]$ vs. $[\mathrm{Fe} / \mathrm{H}]$. Symbols are the same than in Fig. 3.

of $[\mathrm{Ba} / \mathrm{Eu}]=-0.18 \pm 0.09$ slightly higher than the values given by Sneden et al. (1997) for M 15. This ratio is still in the range -0.2 to -0.6 found for field halo stars and globular cluster (M 5, M 13, M 92) giants (Gratton \& Sneden 1991, 1994; Armosky et al. 1994; Shetrone 1996). The [Ba/Eu] ratios found in NGC 6752 are significantly higher than the solar pure r-process ratio. This shows that a part of the $\mathrm{Ba}$ abundance measured in these stars has also an s-process origin.

Strontium is mainly built during the double-shell burning phase of low and intermediate-mass AGB stars, but a significant fraction $(\sim 20 \%)$ could be produced from the He-core burning in massive stars (Raiteri et al. 1993; Prantzos et al. 1990). On the other hand, barium comes mainly from AGB stars in the mass range $\sim 1-4 M_{\odot}$ (Busso et al. 1999). It is therefore interesting to plot the ratio of these two elements as a function of the metallicity. In Fig. 5 we have plotted $[\mathrm{Sr} / \mathrm{Ba}]$ ratios as a function of metallicity for our sample. As the weak s component is of secondary origin at low metallicity, the [Sr/Ba] ratios have to be compared to the solar pure r-process ratio. We have computed the solar $\mathrm{r}$-process fraction $[\mathrm{Sr} / \mathrm{Ba}]_{r}=$ -0.50 , which is displayed in Fig. 5, following Arlandini et al. (1999). A higher ratio is found if we include a weak s-process contribution to the solar $\mathrm{Sr}\left(\left[\mathrm{Sr}_{r+w} / \mathrm{Ba}_{r}\right]=-0.10\right)$. For our sample, we obtain a mean value of $[\mathrm{Sr} / \mathrm{Ba}]=-0.12 \pm 0.21$ which is in good agreement with the ratios found in field halo stars in this metallicity range (e.g. Burris et al. 2000). The subgiants have a lower mean value $(-0.25 \pm 0.07)$ than the TO stars $(0.01 \pm 0.22)$ for the $[\mathrm{Sr} / \mathrm{Ba}]$ ratio, but the scatter in the dwarfs is higher, so that the ratios found in both populations are compatible with an unique mean value. In any case, these ratios show clearly an s-process contribution to the synthesis of the n-capture elements in NGC 6752.

\subsection{Comparison with field stars}

Several studies of barium and europium in Galactic field halo metal-poor stars (McWilliam et al. 1995; McWilliam 1998; Burris et al. 2000; Fulbright 2000) have shown that the $[\mathrm{Ba} / \mathrm{Eu}]$ ratio falls with decreasing metallicity, and approaches a pure r-process ratio in most of the very metal-poor stars.

sample star and are not the simple difference between the mean $[\mathrm{Ba} / \mathrm{Fe}],[\mathrm{Eu} / \mathrm{Fe}]$ and $[\mathrm{Sr} / \mathrm{Fe}]$ ratios. 


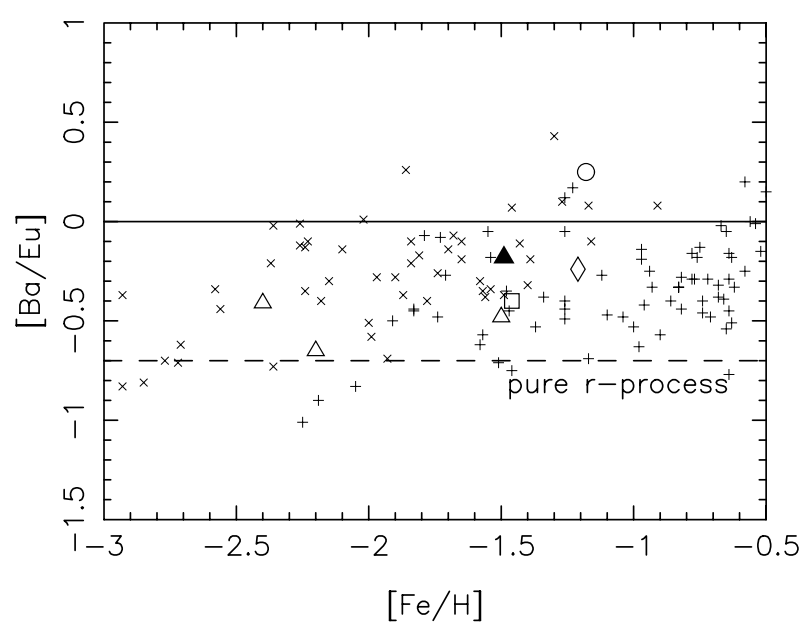

Fig. 6. Comparison of $[\mathrm{Ba} / \mathrm{Eu}]$ ratios for globular clusters and field halo stars. We show here the mean value for our sample stars in NGC 6752 (black triangle). Other clusters are represented by open symbols (with increasing metallicity: M 15, M 92, M 13, M 79, M 5 and M 4). The abundances ratios for globular clusters have been taken in François (1991), Sneden et al. (1997), Ivans et al. (1999), Ivans et al. (2001), and references therein. The field stars (crosses) are from Burris et al. (2000) and Fulbright (2000).

Therefore it is interesting to compare what has been found in globular clusters stars and field halo stars in the same metallicity range.

In Fig. 6 we plot the mean $[\mathrm{Ba} / \mathrm{Eu}]$ ratios for our cluster NGC 6752, and other globular clusters taken in the literature (François 1991; Sneden et al. 1997; Ivans et al. 1999, and references therein). We have added in this figure the data from Burris et al. (2000) and Fulbright (2000) for field stars. Our mean value $[\mathrm{Ba} / \mathrm{Eu}]=-0.18 \pm 0.11$ for a metallicity of $[\mathrm{Fe} / \mathrm{H}]=-1.49 \pm 0.07$ lies between the solar $\mathrm{r}+\mathrm{s}$ mix and $\mathrm{a}$ pure r-process ratio, showing clearly an s-process contribution. Our mean values for $[\mathrm{Ba} / \mathrm{Eu}]$ and $[\mathrm{Sr} / \mathrm{Ba}]$ can also be compared to the much lower values found in the r-element rich stars CS 31082-001 $([\mathrm{Ba} / \mathrm{Eu}]=-0.46,[\mathrm{Sr} / \mathrm{Ba}]=-0.52$, Hill et al. $2002)$ and $\mathrm{CS} 22892-052([\mathrm{Ba} / \mathrm{Eu}]=-0.65,[\mathrm{Sr} / \mathrm{Ba}]=-0.39$, Sneden et al. 2003).

Our data point for NGC 6752 is in good agreement with the field halo stars with the same metallicity. Although the dispersion is rather large, the $[\mathrm{Ba} / \mathrm{Eu}]$ ratios found in globular clusters (Francois 1991; Sneden et al. 1997, 2000; Ivans et al. 1999, 2001; Ramírez \& Cohen 2002, 2003) of different metallicities seem to follow the trend found in halo stars (i.e. $[\mathrm{Ba} / \mathrm{Eu}] \mathrm{de}-$ creasing with decreasing metallicity). However, if we discard the high $[\mathrm{Ba} / \mathrm{Eu}]$ value found in M 4 (Ivans et al. 1999), the data agrees also with a constant value of $\sim-0.4$. It is therefore very important to obtain new data for both the most metal-rich and metal-poor clusters.

\subsection{Self-pollution and self-enrichment of NGC 6752}

The recent results from Gratton et al. (2001) have shown that the $\mathrm{O}-\mathrm{Na}$ and $\mathrm{Mg}-\mathrm{Al}$ anticorrelations found in giant stars of globular clusters are also present in main sequence stars of NGC 6752. These new data can be interpreted in the light of a scenario of self-pollution (Cottrell \& Da Costa 1981). In this scenario, the inhomogeneities are due to the mass lost by intermediate-mass stars during the AGB and planetary nebulae phases. Ventura et al. (2001) performed computations of self-pollution coming from low-metallicity AGBs, and showed that the observed $\mathrm{O}-\mathrm{Na}$ and $\mathrm{Mg}-\mathrm{Al}$ anticorrelations can be explained by the full CNO-cycle operating at the base of the envelope of the most metal-poor models of 4 and $5 M_{\odot}$. In these models, the ${ }^{16} \mathrm{O}$ is reduced and the sodium and aluminium production by proton-capture can occur. These models are only able to explain the anticorrelation of light metals, but not the origin of the metallicity of globular clusters, nor the heavy elements ratios. It is not yet clear whether globular clusters are formed out of matter which is already enriched.

Different authors (Cayrel 1986; Truran et al. 1991; Parmentier et al. 1999) proposed models for the selfenrichment of globular clusters. In these models, a first generation of stars is assumed to form in the central region of the progenitor cloud. When the massive stars of this first generation exploses as SNeII, the matter of the globular cluster is enriched in iron, $\alpha$-elements and heavy elements. If the matter used to form the cluster is of primordial origin, the resulting $[\mathrm{Ba} / \mathrm{Eu}]$ or $[\mathrm{Sr} / \mathrm{Ba}]$ ratios should be compatible with pure r-process values.

Our new data could be used as a test of these scenarii. However, there is no detailed computation concerning the selfenrichement of globular clusters. It is interesting to note that we found a $[\mathrm{Ba} / \mathrm{Eu}]$ and $[\mathrm{Sr} / \mathrm{Ba}]$ ratios departing significantly from the pure r-process ratios, excluding the self-enrichment scenario based only on the contribution of SNeII ejectae. Our values could be explained in the framework of the selfenrichement scenario, but only if an s-process contribution from low-mass AGB stars (Busso et al. 1999) is included. However, the $\mathrm{Ba}$ abundances are constant among the observed stars in NGC 6752, in spite of the large star-to-star variation of the $\mathrm{O}$ and $\mathrm{Na}$ abundances, as found by Gratton et al. (2001). A similar result was found by Armosky et al. (1994) for several other clusters, and later confirmed by other investigators. This indicates that the stars responsible for the O-Na anticorrelation did not produce significant amounts of s-process elements. It is also interesting to remind that we did not find any trend for any of the ratios [n-capture/Fe] as a function of $[\mathrm{Na} / \mathrm{Fe}]$ or $[\mathrm{O} / \mathrm{Fe}]$.

On the other hand, the fact that our abundance ratios are similar to the values found in halo stars with the same metallicity could be interpreted with a model where the globular cluster is formed out of matter which is already enriched in $\mathrm{r}$ - and s-process elements. In the framework of this last scenario, the n-capture elements abundances would have been present at the formation of the cluster, but the dispersion in $\mathrm{O}$ and $\mathrm{Na}$ could still be explained by massive AGBs.

\section{Conclusions}

In this paper, we presented the first abundance determinations for heavy elements in turn-off stars and early subgiants in NGC 6752. These results appear to be the first precise [Sr/Fe], $[\mathrm{Y} / \mathrm{Fe}],[\mathrm{Ba} / \mathrm{Fe}]$, and $[\mathrm{Eu} / \mathrm{Fe}]$ determinations in this cluster. We did not find any large systematic effect between the abundances found in turn-off and subgiant stars (this paper), and giant stars (Norris \& Da Costa 1995). 
We obtain the following mean abundances in our sample (turn-off stars and subgiants):

$[\mathrm{Sr} / \mathrm{Fe}]=0.06 \pm 0.16$

$[\mathrm{Y} / \mathrm{Fe}]=-0.01 \pm 0.12$

$[\mathrm{Ba} / \mathrm{Fe}]=0.18 \pm 0.11$

$[\mathrm{Eu} / \mathrm{Fe}]=0.41 \pm 0.09$.

Our results are in agreement with constant abundance ratios, and the low scatter can be totally explained by uncertainties in their derivation. These ratios are in agreement with the results found in field halo stars with the same metallicity.

We did not observe any correlation between the [n-capture/Fe] ratios and the star-to-star variations of the $\mathrm{O}$ and $\mathrm{Na}$ abundances.

Our mean values $[\mathrm{Ba} / \mathrm{Eu}]=-0.18 \pm 0.11$ and $[\mathrm{Sr} / \mathrm{Ba}]=$ $-0.21 \pm 0.12$ lie both between a pure r-process and the solar $\mathrm{r}+\mathrm{s}$ mix ratios. Looking at these ratios, we showed that NGC 6752 has been polluted by s-process nucleosynthesis. Whether this s-process signature comes from an internal enrichment or is due to a pre-enrichment of the matter from which the cluster formed, remains still an open question.

It would be interesting to extend this kind of analysis to other globular clusters, especially the most metal-rich. Is is only with a significant set of data that the self-enrichement scenario can be tested, although no detailed modelisation has yet been done.

Acknowledgements. The authors would like to thank the referee for his advices and his important contribution to the discussion. G.J. would like to thank R. Cayrel, M. Spite, V. Hill, and E. Depagne (Observatoire de Paris, France), and S. Lefranc (IAS, France) for the many helpful discussions. E.C., S.D., R.G.G., and S.L. research was funded by COFIN 2001028897 by Ministero Universita' e Ricerca Scientifica, Italy.

\section{References}

Alonso, A., Arribas, S., \& Martinez-Roger, C. 1996, A\&A, 313, 873 Arlandini, C., Käppeler, F., Wisshak, K., et al. 1999, ApJ, 525, 886

Armosky, B. J., Sneden, C., Langer, G. E., \& Kraft, R. P. 1994, AJ, 108,1364

Boesgaard, A. M., Deliyannis, C. P., Stephens, A., \& King, J. R. 1998, ApJ, 493, 206

Bonifacio, P., Pasquini, L., Spite, F., et al. 2002, A\&A, 390, 91

Burris, D. L., Pilachowski, C. A., Armandroff, T. E., et al. 2000, ApJ, 544, 302

Busso, M., Gallino, R., \& Wasserburg, G. J. 1999, ARA\&A, 37, 239

Carretta, E., \& Gratton, R. G. 1997, A\&AS, 121, 95

Cayrel, R. 1986, A\&A, 168, 81

Charbonneau, P. 1995, ApJS, 101, 309

Cottrell, P. L., \& Da Costa, G. S. 1981, ApJ, 245, L79

Edvardsson, B., Andersen, J., Gustafsson, B., et al. 1993, A\&A, 275, 101
Edvardsson, B., Gustafsson, B., Johansson, S. G., et al. 1994, A\&A, 290, 176

François, P. 1991, A\&A, 247, 56

Fulbright, J. P. 2000, AJ, 120, 1841

Gratton, R. G., \& Sneden, C. 1991, A\&A, 241, 501

Gratton, R. G., \& Sneden, C. 1994, A\&A, 287, 927

Gratton, R. G., Bonifacio, P., Bragaglia, A., et al. 2001, A\&A, 369, 87

Gratton, R. G., Bragaglia, A., Carretta, E., et al. 2004, A\&A, 408, 529

Grevesse, N., \& Sauval, A. J. 2000, Origin of Elements in the Solar System, Implications of Post-1957 Observations, 261

Grundahl, F., Vandenberg, D. A., Stetson, P. B., Andersen, M. I., \& Briley, M. 2000, The Galactic Halo: From Globular Cluster to Field Stars, 503

Grundahl, F., Briley, M., Nissen, P. E., \& Feltzing, S. 2002, A\&A, 385, L14

Gustafsson, B., Bell, R. A., Eriksson, K., \& Nordlund, A. 1975, A\&A, 42, 407

Hill, V., Plez, B., Cayrel, R., et al. 2002, A\&A, 387, 560

Ivans, I. I., Sneden, C., Kraft, R. P., et al. 1999, AJ, 118, 1273

Ivans, I. I., Kraft, R. P., Sneden, C., et al. 2001, AJ, 122, 1438

King, J. R., Stephens, A., Boesgaard, A. M., \& Deliyannis, C. 1998, AJ, 115, 666

Kraft, R. P., \& Ivans, I. I. 2003, PASP, 115, 143

Lawler, J. E., Wickliffe, M. E., den Hartog, E. A., \& Sneden, C. 2001, ApJ, 563, 1075

Mashonkina, L., Gehren, T., \& Bikmaev, I. 1999, A\&A, 343, 519

Mashonkina, L., \& Gehren, T. 2000, A\&A, 364, 249

Mashonkina, L., \& Gehren, T. 2001, A\&A, 376, 232

Mashonkina, L., Gehren, T., Travaglio, C., \& Borkova, T. 2003, A\&A, 397,275

McWilliam, A., Preston, G. W., Sneden, C., \& Searle, L. 1995, AJ, 109,2757

McWilliam, A. 1998, AJ, 115, 1640

Minniti, D., Geisler, D., Peterson, R. C., \& Claria, J. J. 1993, ApJ, 413, 548

Norris, J. E., \& Da Costa, G. S. 1995, ApJ, 447, 680

Norris, J. E., Da Costa, G. S., \& Tingay, S. J. 1995, ApJS, 99, 637

Parmentier, G., Jehin, E., Magain, P., et al. 1999, A\&A, 352, 138

Prantzos, N., Hashimoto, M., \& Nomoto, K. 1990, A\&A, 234, 211

Raiteri, C. M., Gallino, R., Busso, M., Neuberger, D., \& Kaeppeler, F. 1993, ApJ, 419, 207

Ramírez, S. V., \& Cohen, J. G. 2002, AJ, 123, 3277

Ramírez, S. V., \& Cohen, J. G. 2003, AJ, 125, 224

Ryan, S. G., Norris, J. E., \& Bessell, M. S. 1991, AJ, 102, 303

Shetrone, M. D. 1996, AJ, 112, 1517

Sneden, C., McWilliam, A., Preston, G. W., et al. 1996, ApJ, 467, 819

Sneden, C., Kraft, R. P., Shetrone, M. D., et al. 1997, AJ, 114, 1964

Sneden, C., Pilachowski, C. A., \& Kraft, R. P. 2000, AJ, 120, 1351

Sneden, C., Cowan, J. J., Lawler, J. E., et al. 2003, ApJ, 591, 936

Spite, M. 1967, Ann. Astrophys., 30, 211

Truran, J. W., Brown, J., \& Burkert, A. 1991, The Formation and Evolution of Star Clusters, ASP Conf. Ser., 13, 78

Ventura, P., D’Antona, F., Mazzitelli, I., \& Gratton, R. 2001, ApJ, 550, L65

Yong, D., Grundahl, F., Lambert, D. L., Nissen, P. E., \& Shetrone, M. D. 2003, A\&A, 402, 985

Zinn, R., \& West, M. J. 1984, ApJS, 55, 45 